\title{
Paracoccus yeei
}

National Cancer Institute

\section{Source}

National Cancer Institute. Paracoccus yeei. NCI Thesaurus. Code C123537.

A species of aerobic Gram-negative coccobacillus in the family Rhodobacteraceae. This species is catalase and oxidase positive and can reduce nitrate. P. yeei is found naturally in soil and brines and has be identified as an unusual etiologic opportunistic agent of peritonitis. 